\title{
Gastric-Type Extremely Well-Differentiated Adenocarcinoma of the Stomach: A Challenge for Preoperative Diagnosis
}

\author{
Mee Joo · Song Hee Han \\ Department of Pathology, Inje University \\ Ilsan Paik Hospital, Goyang, Korea \\ Received: June 18, 2015 \\ Revised: July 10, 2015 \\ Accepted: July 14, 2015 \\ Corresponding Author \\ Mee Joo, MD, PhD \\ Department of Pathology, Inje University \\ Ilsan Paik Hospital, 170 Juhwa-ro, Ilsanseo-gu, \\ Goyang 10380, Korea \\ Tel: +82-31-910-7141 \\ Fax: +82-31-910-7139 \\ E-mail:mjoo@paik.ac.kr
}

\begin{abstract}
Gastric-type extremely well-differentiated adenocarcinoma (EWDA) is a rare type of gastric adenocarcinoma characterized by infiltration of well-formed mucinous glands with little or no nuclear atypia, which resemble foveolar epithelium or pyloric glands. Because of its high degree of differentiation, preoperative biopsy diagnosis of gastric-type EWDA is very difficult. We encountered a case of gastric-type EWDA, manifesting as a Borrmann type 4 lesion, in a 47-year-old man. Despite four repeated biopsies, the preoperative biopsy diagnosis was not conclusive due to the scarcity of diagnostic tumor cells and lack of knowledge regarding the unusual histologic findings of gastric-type EWDA. We herein describe the histologic findings of gastric-type EWDA in detail, with the aim of facilitating a preoperative biopsy diagnosis and understanding of this rare type of gastric adenocarcinoma.
\end{abstract}

Key Words: Stomach neoplasms; Extremely well-differentiated adenocarcinoma; Gastric-type
Extremely well-differentiated adenocarcinoma (EWDA) is a rare type of gastric adenocarcinoma, which accounts for less than $0.2 \%$ of all gastric cancers. ${ }^{1}$ EWDA, as its name suggests, is highly differentiated and almost indistinguishable from regenerating atypia or inflammatory changes. Such a high degree of differentiation and minimal cellular atypia commonly lead to diagnostic difficulties, particularly with preoperative forceps biopsy. ${ }^{1,2}$ Based on histological and immunohistochemical differences, EWDAs can be classified into two variants: intestinaltype and gastric-type. ${ }^{2}$ In 1999, Endoh et al. ${ }^{3}$ first coined the term "extremely well differentiated adenocarcinoma of the stomach" to describe well-differentiated adenocarcinoma with low-grade nuclear atypia and morphology mimicking intestinal metaplasia, which is currently considered a prototype of intestinal-type EWDA. Meanwhile, gastric-type EWDAs are quite different from intestinal-type EWDAs, which consist of benignlooking mucous cells resembling foveolar epithelium, mucus neck cells, or pyloric glands and contain gastric phenotype mucin. $^{2,45}$ The frequency of diagnosing intestinal-type EWDA with biopsy specimens prior to resection is increasing, since characteristic architectural features of the neoplastic glands, which resemble the shape of the letter $\mathrm{W}, \mathrm{H}, \mathrm{Y}$, or $\mathrm{X}$, have been reported to be the most informative and useful diagnosis criteria. ${ }^{6}$ How- ever, since little is known about gastric-type EWDA due to its rarity, its preoperative diagnosis with biopsy remains challenging. The present case emphasizes the importance of being aware of this rare type of adenocarcinoma in order to make a preoperative biopsy diagnosis.

\section{CASE REPORT}

A 47-year-old man presented with epigastric discomfort and dyspepsia. Esophagogastroduodenoscopy revealed a diffusely infiltrative submucosal lesion involving the upper body of the stomach along the greater curvature (Fig. 1A). The overlying mucosa was intact and non-ulcerative, but it showed tiny scattered mucosal openings with extruding mucus (Fig. 1B). Abdominal computed tomography scan demonstrated diffuse wall thickening with suspicious perigastric infiltration, consistent with Borrmann type 4 gastric cancer. The patient underwent four consecutive gastrofiberscopic examinations, with biopsies consisting of more than 10 pieces, but all biopsies were negative for adenocarcinoma. Subsequently, the patient underwent a diagnostic laparotomy and total gastrectomy and was diagnosed with gastric-type EWDA (pT4aN3aM1, stage IV). Microscopic examination revealed numerous irregular-shaped mucinous 
glands that diffusely extended from the submucosal layer to the visceral peritoneum (Fig. 1C). The mucosal layer was spared for the most part, with only some carcinoma glands found in the deep portion of the mucosa. The tumor glands consisted of mucin-rich columnar cells with basally located, small, bland-looking nuclei mimicking hyperplastic foveolar epithelium or dilated pyloric glands (Fig. 1D), which deeply infiltrated the proper muscle with no desmoplastic stromal reaction (Fig. 1E). There were also areas showing distinctly lobular proliferation of small glands often surrounding a larger central duct (Fig. 1F); such areas were frequently observed in the submucosa, and the cen- tral dilated duct occasionally opened through the overlying mucosal surface (Fig. 1G). The tumor cells were diffusely positive for MUC5AC (Fig. 1H), human gastric mucin (HGM), and carcinoembryonic antigen (CEA); focally positive for MUC6; and negative for MUC2, CD10, and p53. The Ki-67 labeling index was low $(5 \%)$. We retrospectively reviewed the preoperative biopsy specimens and found a few scattered carcinoma glands in the basal portion of the oxyntic-type corpus mucosa (Fig. 2A). Despite not being atypical enough to be considered definite carcinoma, the glands were indisputably noticeable, with large size, abundant clear cytoplasm, mild nuclear atypia, and unusual
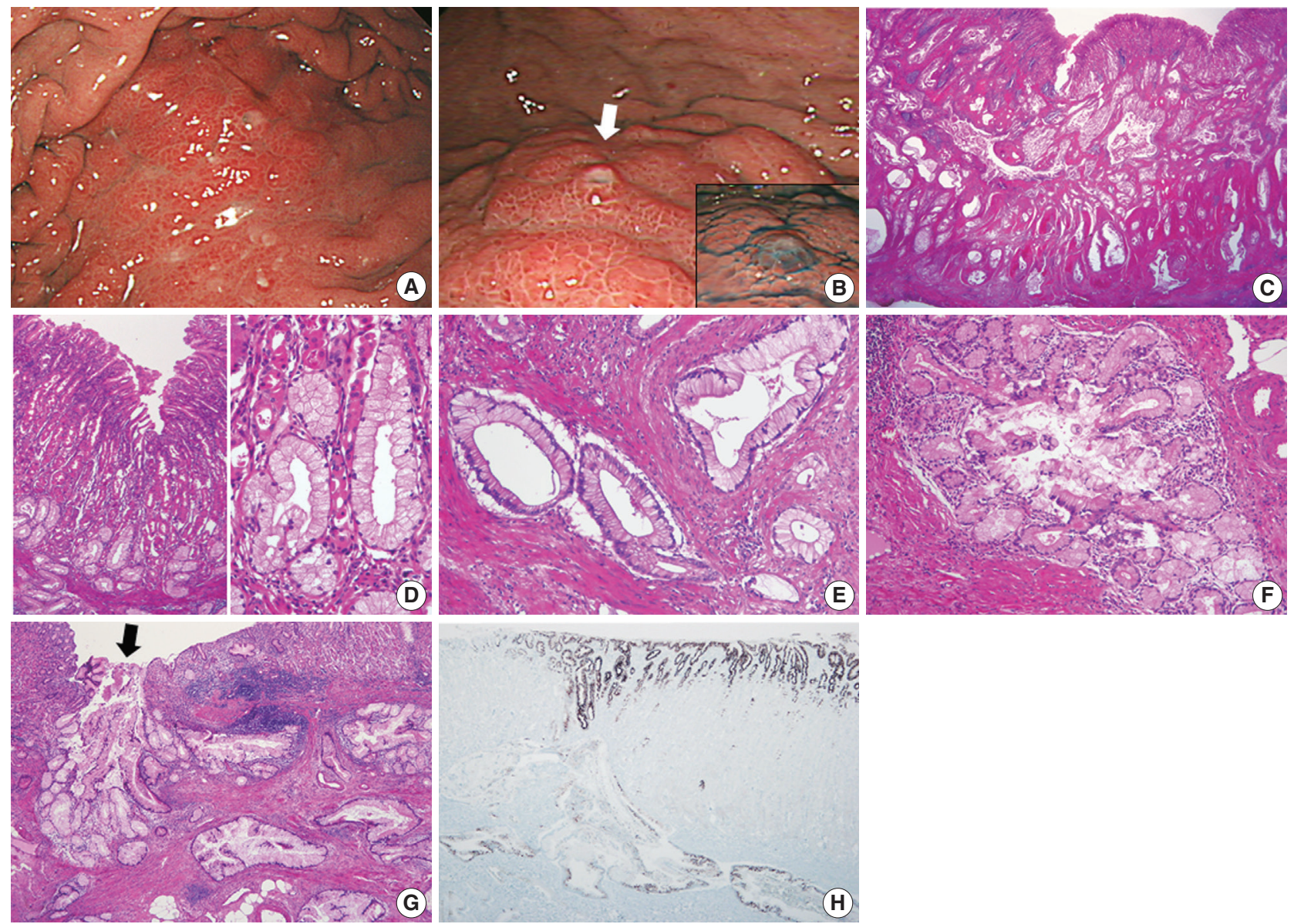

Fig. 1. (A, B) Esophagogastroduodenoscopy. (A) A submucosal infiltrative lesion is observed in the upper body. (B) The overlying mucosa is mainly intact with scattered small openings with protruding mucus (arrow and inset). (C-E) Representative microphotographs of invasive carcinoma. (C) Low-magnification view demonstrates that the entire gastric wall is infiltrated by irregular neoplastic glands showing frequent cystic dilatation and intraluminal mucinous material. (D) The mucosal surface is mainly intact, and the neoplastic glands partly involve the deep portion of the mucosa. The neoplastic glands with basally-located, small, bland nuclei and abundant mucin-containing cytoplasm mimic normal gastric foveolar epithelium or pyloric glands. Compared to the adjacent normal glands, they are slightly larger in size and more irregular in shape. (E) Infiltrating neoplastic glands show no significant cytological or architectural atypia and no desmoplastic stromal reaction. $(F, G)$ Representative microphotographs of lobular endocervical glandular hyperplasia-like areas. (F) A cluster of small glands surrounds a central large duct in a distinct lobular arrangement with a sharp border. (G) Such areas are mostly observed in the submucosal layer, and occasionally a central dilated duct with mucinous materials opens through the overlying mucosa (arrow), which is in concordance with mucus protrusion on endoscopy (arrow in Fig. 1B). (H) Immunostaining for MUC5AC is diffusely positive in the tumor glands and foveolar epithelial cells. 
location compared to normal mucous glands (Fig. 2B, C). Those atypical glands were more distinguishable on immunostaining for HGM, CEA, and MUC5AC (Fig. 2D).

\section{DISCUSSION}

To date, only nine cases (including the present one) of gastrictype EWDA have been reported in the English literature. The clinical and pathological findings of these patients are summa- rized in Table 1. The patients were all male with ages ranging from 47 to 81 years. The tumors in all reported cases were located in the upper or middle third of the stomach. Grossly, they were polypoid masses or submucosal tumors mimicking Borrmann type 4 gastric cancer. Preoperative biopsy findings were available in seven cases, five of which were diagnosed as benign lesions, one as a suspicious carcinoma, and one as definite carcinoma on repeat biopsy. It is very difficult to diagnose gastrictype EWDA in a preoperative biopsy specimen, especially in
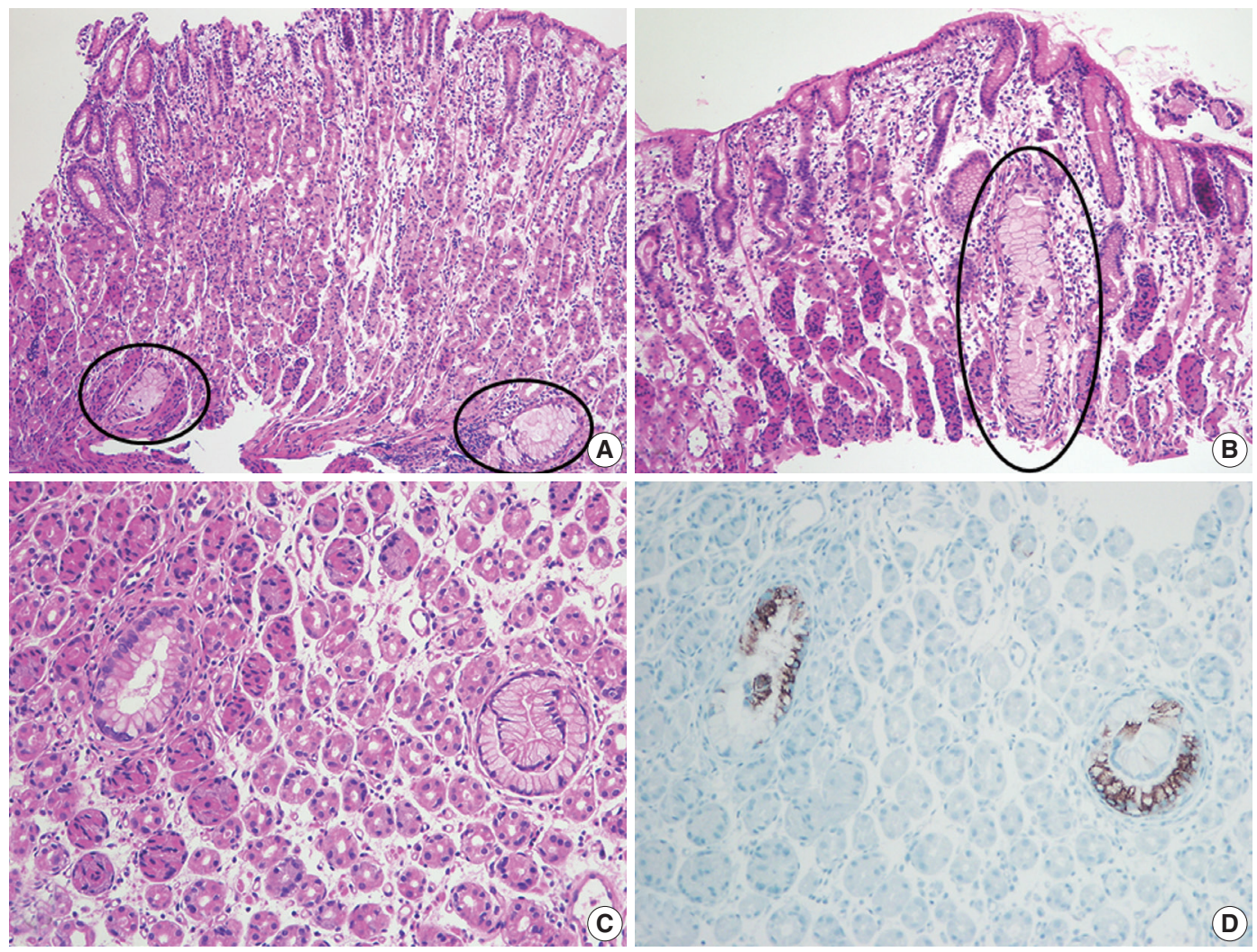

Fig. 2. Preoperative biopsy findings. (A, B) There are a few atypical mucinous glands (circles) that are noticeably large in size and observe frequently in the basal portion of the corpus mucosa. (C) Such mucinous glands display mild nuclear atypia, but they are not atypical enough to be recognized as adenocarcinoma. (D) These glands are highlighted by MUC5AC immunostaining.

Table 1. Clinical and pathological findings of previously published cases of gastric-type EWDA in the English literature

\begin{tabular}{|c|c|c|c|c|c|c|c|}
\hline $\begin{array}{l}\text { Case } \\
\text { No. }\end{array}$ & Reference & Sex/Age (yr) & Location & $\begin{array}{l}\text { Size of tumor } \\
(\mathrm{cm})\end{array}$ & Macroscopic type & $\begin{array}{l}\text { Depth of } \\
\text { invasion }\end{array}$ & $\begin{array}{l}\text { Preoperative biopsy } \\
\text { diagnosis }\end{array}$ \\
\hline 1 & Niimi et al. ${ }^{1}$ & $M / 58$ & Upper third & 7 & Borrmann type 4 & T3 & Benign \\
\hline 2 & Yao et al. ${ }^{2}$ & $M / 81$ & Middle third & 5.5 & EGC type 0-I & $\mathrm{T} 1 \mathrm{~b}$ & Benign \\
\hline 3 & Yao et al. ${ }^{2}$ & $M / 51$ & Upper third & 2.5 & EGC type 0 -lla & $\mathrm{T} 1 \mathrm{~b}$ & NA \\
\hline 4 & Yao et al. ${ }^{2}$ & $M / 63$ & Middle third & 8 & Borrmann type 1 & T3 & Benign \\
\hline 5 & Yao et al. ${ }^{2}$ & $\mathrm{M} / 76$ & Upper third & 3.5 & Borrmann type 1 & T3 & Suspicious for CA \\
\hline 6 & Yao et $a l^{2}$ & $M / 57$ & Upper third & 5 & Borrmann type 1 & T3 & NA \\
\hline 7 & Nokubi et al. ${ }^{4}$ & $\mathrm{M} / 60$ & Cardia & NS & Borrmann type 4 & $\mathrm{~T} 4 \mathrm{~b}$ & CA on repeated biopsy \\
\hline 8 & $\mathrm{Lee}^{5}$ & $\mathrm{M} / 67$ & Cardia & 7 & Borrmann type 1 & T3 & Benign \\
\hline 9 & Present case & $\mathrm{M} / 47$ & Upper third & 6.5 & Borrmann type 4 & $\mathrm{~T} 4 \mathrm{a}$ & Benign \\
\hline
\end{tabular}

EWDA, extremely well-differentiated adenocarcinoma; M, male; EGC, early gastric cancer; NA, not assessed; CA, carcinoma; NS, not stated. 
patients with Borrmann type 4 lesions. Nevertheless, the most important aspect in diagnosing gastric-type EWDA in a biopsy specimen is observation of the subtle atypical changes of mucous glands: mucous glands with conspicuously abundant mucinous cytoplasm, mild nuclear atypia, and slightly larger size should raise suspicion of gastric-type EWDA, particularly when they are abnormally clustered or seen in the deep portion of the corpus mucosa that is usually devoid of well-formed mucous glands. Additional immunostaining for CEA and gastric phenotype mucin can help to confirm the diagnosis.

The histologic characteristics of gastric-type EWDA, including deep invasion of extremely well-formed mucinous glands, minimal cellular atypia, and no significant desmoplastic stromal reaction, resemble those of minimal deviation adenocarcinoma (MDA) of the uterine cervix. Therefore, gastric-type EWDA has been considered as the gastric counterpart of MDA.,5 According to the new World Health Organization classification published in 2014, MDA is classified as an extremely well-differentiated form of gastric-type endocervical mucinous adenocarcinoma. ${ }^{7}$ Lobular endocervical glandular hyperplasia (LEGH) has been described as a distinctive hyperplastic lesion of the endocervix characterized by lobular proliferation of small, rounded, noninvasive glands often surrounding a central dilated gland. It has been postulated that LEGH is a precursor lesion of MDA. ${ }^{8,9}$ In the present case, we found LEGH-like areas with distinct histological characteristics of LEGH that occurred concurrently with invasive mucinous carcinoma. In addition, they were predominantly observed in the submucosal layer, just as LEGH is usually confined to the inner half of the endocervical wall. LEGHlike lesions have never been described in the stomach before, which may provide evidence to support gastric-type EWDA being the gastric counterpart of MDA of the uterine cervix.

\section{Conflicts of Interest}

No potential conflict of interest relevant to this article was reported.

\section{REFERENCES}

1. Niimi C, Goto H, Ohmiya N, et al. Usefulness of p53 and Ki-67 immunohistochemical analysis for preoperative diagnosis of extremely well-differentiated gastric adenocarcinoma. Am J Clin Pathol 2002; 118: 683-92.

2. Yao T, Utsunomiya T, Oya M, Nishiyama K, Tsuneyoshi M. Extremely well-differentiated adenocarcinoma of the stomach: clinicopathological and immunohistochemical features. World J Gastroenterol 2006; 12: 2510-6.

3. Endoh Y, Tamura G, Motoyama T, Ajioka Y, Watanabe H. Well-differentiated adenocarcinoma mimicking complete-type intestinal metaplasia in the stomach. Hum Pathol 1999; 30: 826-32.

4. Nokubi M, Kawanowa K, Kawata H, Hanatsuka K, Hosoya Y. Extremely well-differentiated adenocarcinoma of the gastric cardia: a unique case with columnar cells and laminated stones. Pathol Int 2004; 54: 854-60.

5. Lee WA. Gastric extremely well differentiated adenocarcinoma of gastric phenotype: as a gastric counterpart of adenoma malignum of the uterine cervix. World J Surg Oncol 2005; 3: 28.

6. Ushiku T, Arnason T, Ban S, et al. Very well-differentiated gastric carcinoma of intestinal type: analysis of diagnostic criteria. Mod Pathol 2013; 26: 1620-31.

7. Kurman RJ, Carcangiu ML, Herrington CS, Young RH. WHO classification of tumors of female reproductive organs. 4th ed. Lyon: International Agency for Research on Cancer, 2014; 185-6.

8. Mikami Y, McCluggage WG. Endocervical glandular lesions exhibiting gastric differentiation: an emerging spectrum of benign, premalignant, and malignant lesions. Adv Anat Pathol 2013; 20: 227-37.

9. Kawauchi S, Kusuda T, Liu XP, et al. Is lobular endocervical glandular hyperplasia a cancerous precursor of minimal deviation adenocarcinoma?: a comparative molecular-genetic and immunohistochemical study. Am J Surg Pathol 2008; 32: 1807-15. 